\title{
EDITORIALS
}

\section{Protecting the Public: Is It Time for a Paradigm Shift in Expected Practice Standards?}

\author{
Robyn Tamblyn, $P h D^{1,2}$ \\ 'Department of Medicine, McGill University, Montreal, QC, Canada; ${ }^{2}$ Department of Epidemiology and Biostatistics, James McGill Chair, \\ McGill University, Montreal, QC, Canada.
}

$\mathrm{J}$ Gen Intern Med 26(5):460-1

DOI: $10.1007 / \mathrm{s} 11606-011-1644-6$

() Society of General Internal Medicine 2011

$\mathrm{C}$ redentialing processes, such as certification, are used to assure the public that licensed health professionals will deliver safe and effective health care $^{1}$. Methods to ensure safe and effective practitioners have traditionally focused on training and assessment of competence before entering practice. However, an increasing number of visionary credentialing bodies such as the American Board of Internal Medicine have created systematic methods of measuring a practitioner's ability to deliver care in practice ${ }^{2}$.

Although new methods for monitoring practice have been developed for quality improvement and pay for performance, in this issue of JGIM, Hess and colleagues are the first to take on the challenge of establishing benchmarks for performance in practice to assure the public that their certificants offer safe and effective care ${ }^{2}$. Indeed, Hess creatively translates methods developed for setting passing standards in licensing and certifying examinations to an approach that could be used to make judgments about the adequacy of clinical practice.

The rigor of Hess's research is beyond reproach. However, there are two important assumptions of the Hess paradigm that need to be challenged and rigorously debated. To set the stage, I have depicted public expectations of safe and effective care within an outcome-based quality framework ${ }^{3}$ (Fig. 1).

A woman who has, for example, diabetes would expect that over time (X axis) she would achieve the optimal health status (Y axis) possible for her condition based on current advances in science on prevention and treatment (A), recognizing that optimal outcomes may be attenuated by the resources available in the health system to provide this level of care (B). She would expect that care provided would not result in outcomes that were worse than the natural course of illness (C) and

Published online February 12, 2011 would avoid problems created by potentially preventable errors and adverse effects (D).

The first assumption I would challenge in the Hess paradigm is the assessment of performance based on expert opinion about the relevance of care processes. If the public is to be protected, performance needs to be measured in relationship to optimal health outcomes (A), perhaps conditioned by judgment or empirical evidence of what is possible within resource constraints (B). For common chronic conditions such as cardiovascular disease ${ }^{4}$, existing statistical models can be used to predict optimal achievable outcomes. Based on longitudinal cohort studies and randomized trials, empirical data are available to estimate the impact on health outcomes of both non-modifiable characteristics (such as age and sex) and risk factors that can be modified by medical intervention (e.g., smoking, lipid levels, glucose control). The goal for each patient, and for all patients in the practice of each physician, is to achieve optimal outcomes within the constraints of non-modifiable characteristics such as age, sex, race, and eventually genotype. The quality of a physician's performance is then a direct function of the extent to which his/her patients reach their expected health status, achieved through successful modification of known risk factors. Moreover, utilization of an outcome framework provides data that can directly measure the effectiveness of physicians in achieving health outcome targets for the population, and identify suboptimal or variable aspects of management that could be targeted for professional development and policy intervention. Indeed, physicians are already using predictive statistical models in practice to calculate risk, assess prognosis, and assist patients in reducing their risk of adverse events ${ }^{5,6}$.

The second assumption that should be debated is the primary goal of the maintenance of certification program (MOC). Is the goal to identify the bottom $5 \%$ of physicians who may deliver unsafe or ineffective care? If this were the primary goal, different outcomes would need to be measured. Specifically, the rate of potentially preventable adverse events (Fig. 2; slope D) would need to be assessed, such as the rate of hospitalizations for avoidable deterioration in disease status or death due to problems such as 


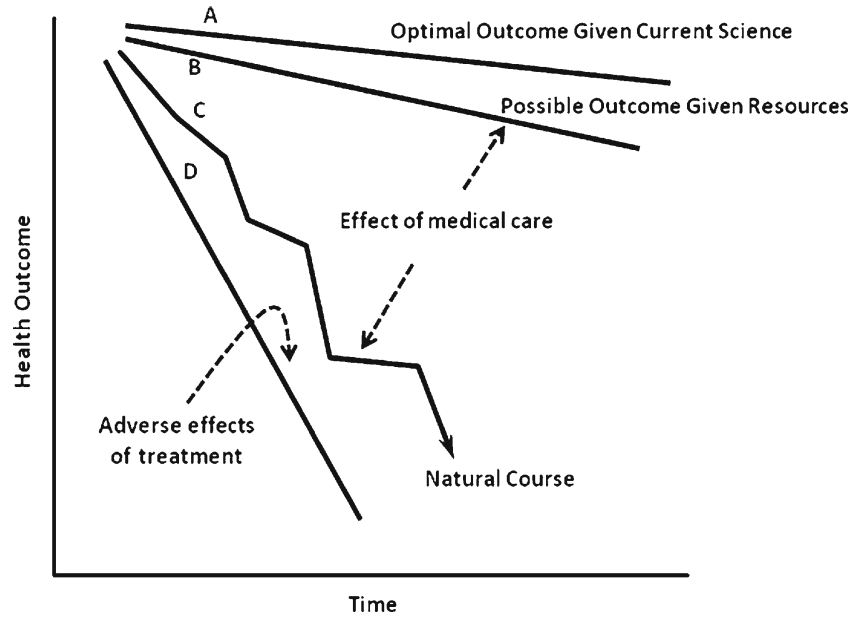

Figure 1. Outcome framework for performance assessment.

inadequate prevention, intervention and follow-up. While monitoring catastrophic rates of adverse outcomes, such as those seen in the Bristol cases ${ }^{7}$, is warranted, I suggest that the main goal of MOC should be to enhance the overall level of performance of all certificants and narrow the variation in outcomes that patients might expect between practicing physicians (e.g., Fig. 2).

No patient will want to achieve $49 \%$ of his/her optimal outcome with Physician A when Physician B in the same clinic or city can achieve $98 \%$. To achieve better outcomes for the majority of patients, MOC programs need to target professional enhancement to achieve better outcomes among all physicians, not just the $5 \%$ at the lower end of the distribution.

In this century, there is a call for rigorous approaches to improve accountability, safety, and effectiveness in health care. To address this challenge, the profession needs to define the best model for monitoring and enhancing safety and quality in practice, and engage medical leadership to turn the best of all ideas into action.

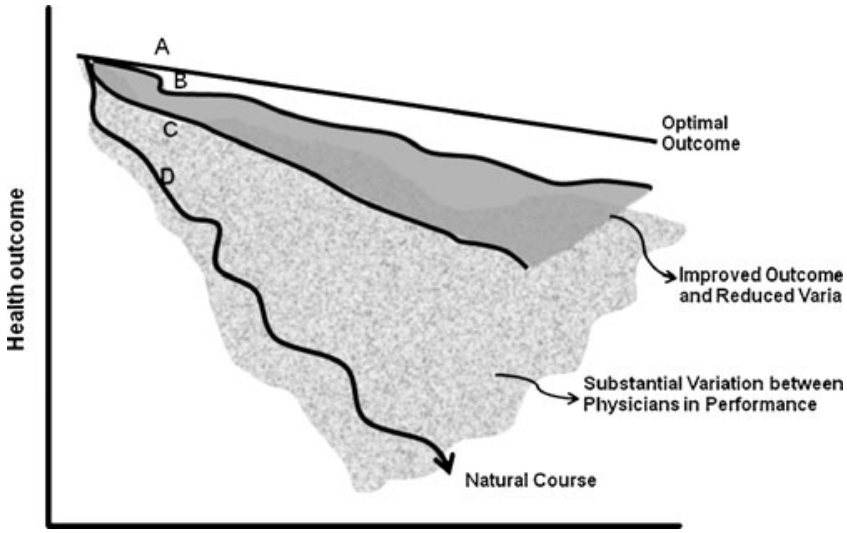

Figure 2. Reducing variation in performance.

Corresponding Author: Robyn Tamblyn, PhD; Department of Medicine, McGill University, 1140 Pine Ave. West, Montreal, BC H3A 1A3, Canada (e-mail: robyn.tamblyn@mcgill.ca).

\section{REFERENCES}

1. Tamblyn RM. Is the public being protected? Prevention of suboptimal medical practice through training programs and credentialing examinations. Eval Health Prof 1994;17(2):198-221.

2. Hess BJ, Weng W, Lynn LA, Holmboe ES, Lipner RS. Setting a fair performance standard for physicians' quality of patient care. J Gen Intern Med 2011; doi:10.1007/s11606-010-1572-x.

3. Tamblyn R, Dauphinee D. Outcomes in medical education: What is the standard and outcome of care delivered by our graduates? Advances in Health Sciences Education 1999;4(1):9-25.

4. Conroy RM, Pyorala K, Fitzgerald AP et al. Estimation of ten-year risk of fatal cardiovascular disease in Europe: the SCORE project. Eur Heart J 2003;24(11):987-1003

5. Altman DG, Vergouwe Y, Royston P, Moons KG. Prognosis and prognostic research: validating a prognostic model. Br Med J 2009;338:b605.

6. Lowensteyn I, Joseph L, Levinton C, Abrahamowicz M, Steinert Y, Grover S. Can computerized risk profiles help patients improve their coronary risk? The results of the Coronary Health Assessment Study (CHAS). Prev Med 1998;27(5 Pt 1):730-737.

7. The Bristol Royal Infirmary Inquiry. The Report of the Public Inquiry into children's heart surgery at the Bristol Royal Infirmary 1984-1995Learning from Bristol. London, UK: The Bristol Royal Infirmary Inquiry; 2001. Report No.: CM 5207. 